4. Oyama, H. Ryoanji Sekitei: Nanatsu no Nazo wo toku (Ryoanji Rock Garden: Resolving Seven Mysteries) (Kodansha, Tokyo, 1995).

5. Kovacs, I. \& Julesz, B. Nature 370, 644-646 (1994).

6. Leyton, M. Comp. Vis. Graph. Image Proc. 38, 327-341 (1987).

COMMUNICATIONS ARISING

Fruitflies

\section{Pigmentation and mate choice in Drosophila}

1 any species of the fruitfly Drosophila are either sexually dimorphic for abdominal pigmentation (the posterior segments in males are black and those of females have thin dark stripes) or sexually monomorphic for this pigmentation (both sexes show striping). Kopp et al. ${ }^{1}$ report a correlation in two Drosophila clades between the expression of the bric-à-brac (bab) gene, which represses male-specific pigmentation in D. melanogaster females, and the presence of sexually dimorphic pigmentation. They suggest that sexual selection acted to produce sexual dichromatism in Drosophila by altering the regulation of $b a b$, on the grounds that $D$. melanogaster males show a strong mate preference for females with lightly pigmented abdomens, and that this discrimination helps to maintain sexual dichromatism by preventing males from wasting time by courting other (darkly pigmented) males. Here we show that the mate discrimination observed by Kopp et al. ${ }^{1}$ may in fact have resulted from the nature of the strains and comparisons they used in their study and so could be irrelevant to mate choice in nature.

Kopp et al. did not record the specific pairs of female strains used in their 'light versus dark' comparisons (A. Kopp, personal communication), so we could not repeat their experiments exactly. They did, however, use inbred stocks or genetic strains that were not controlled for their genetic background, so that mate choice could be affected by many factors besides pigmentation. We carried out two sets of experiments in which we eliminated this possibility by using females with homogeneous genetic backgrounds derived from the wild. In contrast to Kopp et $a l .{ }^{1}$, we found no evidence that males choose less-pigmented females.

We replicated Kopp and colleagues' methods ${ }^{1}$ by placing one wild-type male in a vial containing two virgin females that had different degrees of abdominal pigmentation (all flies were 4 days old), and observing each pair for $30 \mathrm{~min}$. In all vials in which matings occurred, we scored the degree of pigmentation of the A5 and A6 abdominal segments of mated and unmated females using the procedure described by David et $a l^{2}$. This method generates pigmentation scores ranging from zero (no pigmentation) to 20 (both segments 100\% pigmented).

In our first experiment, we compared two
7. Taylor, R. P. Nature 415, 961 (2002).

8. Taylor, R. P., Micolich, A. P. \& Jonas, D. Nature 399, 422 (1999) Supplementary information accompanies this communication on Nature's website.

Competing financial interests: declared none.

types of female: those with wild-type $b a b$ function (normal, light pigmentation) and those with only one functional $b a b$ copy $\left(b a b^{-} / b a b^{+}\right.$heterozygotes; darker, male-like pigmentation). Chromosomes either containing or lacking the $b a b$ locus were placed in a wild-type genetic background derived from a $D$. melanogaster stock founded by females collected during 2000 in Arkansas and Louisiana ('ArkLa'). Dark and light females were respectively produced by mating ArkLa males with females from two deficiency strains, Df(3L)Ar12-1 and Df(3L)Ar11. (The former strain was also used by Kopp et al.) Both deficiencies are similar in size and were created in the same genetic background, but Df(3L)Ar12-1 deletes the $b a b$ locus, producing dark heterozygous females (average pigmentation score, 16.4 \pm 0.09 (s.e.)), whereas females heterozygous for $\operatorname{Df}(3 \mathrm{~L}) \operatorname{Ar} 11$, which does not delete the $b a b$ locus, are lighter (average score, 11.2 \pm 0.15 ).

ArkLa males that were given a choice between $b a b^{-}$and $b a b^{+}$heterozygous females did not discriminate between these types (94 'dark' matings, 88 'light'; $\chi^{2}=0.2$, $P=0.67)$. These results differ significantly $\left(G=38.3, P<1 \times 10^{-9}\right)$ from the combined results of Kopp et al. ${ }^{1}$, who observed 23 'dark' and 105 'light' matings.

In our second experiment, we produced females of varying pigmentation in the $F_{2}$ generation of a cross between an outbred stock of D. melanogaster collected in Winters, California, during 2000 and a 'light' female stock produced by combining two inbred lines from the same locality and collected in 2000 (S. Nuzhdin). Males from the outbred stock were given a choice between dark and light $\mathrm{F}_{2}$ females, with mean pigmentation scores of $11.9 \pm 0.17$ and $7.5 \pm 0.24$, respectively. Again, males showed no significant discrimination between dark and light females (81 'dark' matings, 61 'light'; $\left.\chi^{2}=2.82, P=0.095\right)$.

Our two replicate experiments were statistically homogeneous ( $G=0.94, P=0.33$ ), but our combined data differed significantly from those of Kopp et al. $(G=52.0$, $\left.P<1 \times 10^{-10}\right)$. Far from showing a strong preference for light females, our wild-type males showed an insignificant tendency to mate with darker females.

We suggest that Kopp and colleagues' results may be attributed to their comparing mutant or inbred strains with dissimilar genetic backgrounds, so that 'light' and 'dark' females in each trial differed in many of their genes. This idea is supported by the extraordinarily high proportion of trials observed by Kopp et al. in which neither female mated (42 out of 170, 24.7\%; A. Kopp, personal communication), compared with the low proportion of such trials in our experiments ( 14 out of $324,4.3 \%$ ). This difference is highly significant $(G=43.8$, $\left.P<1 \times 10^{-10}\right)$. Although sexual selection may account for the differences in pigmentation among Drosophila species, we find no evidence that it operates in D. melanogaster in the way suggested by Kopp et al.

Anna Llopart, Susannah Elwyn,

Jerry A. Coyne

Department of Ecology and Evolution, University of Chicago, Chicago, Illinois 60637, USA

e-mail: j-coyne@uchicago.edu

1. Kopp, A., Duncan, I. \& Carroll, S. B. Nature 408, 553-559 (2000); correction, Nature 410, 611 (2001).

2. David, J. R., Capy, P., Payant, V. \& Tsakas, S. Gen. Sel. Evol. 17, 211-224 (1985).

Kopp et al. reply - To appreciate how new morphological traits arise in the course of evolution, we need to understand both the genetic basis of phenotypic changes and the selective forces that promote them. We presented evidence that evolutionary changes in the regulation of the $b a b$ gene could account for the origin of sexually dimorphic abdominal pigmentation in D. melanogaster; we also investigated whether sexual selection could explain the origin and maintenance of this trait.

We found that, given a choice between wild-type and bab-mutant females (which have ectopic male-like pigmentation), $D$. melanogaster males discriminated in favour of normally pigmented females. This effect was observed in several combinations of bab-mutant and wild-type strains, but was abolished when white-mutant males, which are effectively blind, were used in matechoice experiments. On this basis, we suggested that sexual selection against darkly pigmented females can account for the maintenance of sexual dimorphism.

However, Llopart et al. argue that this mechanism is unlikely to operate in nature. The difference between our findings is presumably due to the choice of model fly strains. As Llopart et al. point out, both the males and females used in our experiments were derived from highly inbred laboratory strains, and extrapolation to natural populations seems not to be supported.

The questions remain - why did male-specific pigmentation evolve in $D$. melanogaster but not in other Drosophila lineages? Why is it absent in females? And what selective pressure has maintained this dimorphism for over 20 million years? For now, the answers are that we do not know. Artyom Kopp, Sean B. Carroll

Howard Hughes Medical Institute and Laboratory of Molecular Biology, University of WisconsinMadison, Madison, Wisconsin 53706-1596, USA

e-mail:sbcarrol@facstaff.wisc.edu 